\title{
FUSE observations of the SMC Wolf-Rayet binary Sand 1 (WO4+O4V): atmospheric eclipses and colliding winds
}

\author{
Nicole St-Louis \& Anthony F.J. Moffat \\ Département de physique, Université de Montréal, \\ C.P. 6128, Succ. Centre-Ville, Montréal, QC H3C 3J7, Canada, and \\ Observatoire du mont Mégantic, Canada
}

Sergey V. Marchenko

Department of Physics and Astronomy, Western Kentucky University, 1 Big Red Way, Bowling Green, KY 42101-3576, USA

\begin{abstract}
We present FUSE observations of the SMC WO4+O4V binary Sand 1. Our spectra show variations in S IV, C III, CIV and O VI lines, which we attribute mainly to emission from the shock cone, resulting from the collision between the two winds, and to atmospheric eclipses of the O-star continuum light by the WR wind. From this variability, we deduce a cone opening angle of $120^{\circ}$.
\end{abstract}

\section{When two mighty winds meet}

When the winds of two hot, massive stars in a binary interact, two main phenomena occur: ( $i)$ a shock-cone forms where the momentum fluxes of the winds are equal. As the heated gas flows along the shock, it cools and emits light in lines of gradually decreasing ionisation potential; $(i i)$ the WR wind absorbs the O-star light in certain lines. This is the so-called atmospheric eclipse. The shape of the absorption is determined by the wind density and velocity encountered by the light. Comparing the spectral variability generated by these phenomena with models, allows one to constrain various wind and orbital parameters.

\section{The observations}

We have secured 18 FUSE spectra of the WO4+O4V binary Sand 1 and retrieved 4 archival spectra. The lines that dominate the spectrum are formed in the WR wind: S IV $\lambda$ 933/945, C III $\lambda 977$, O vi $\lambda 1032 / 1038$ and $\mathrm{C}$ IV $+\mathrm{CIII} \lambda 1168+\lambda 1175$. This binary has a circular orbit with a period of $16.64 \mathrm{~d}$ and an orbital inclination of $68^{\circ}$ (obtained from polarisation data of one of us, AFJM).

\section{Some results}

Close inspection of the spectra reveals similar variations in the S IV, C III $\lambda 977$ and $\mathrm{O}$ VI lines. Excess emission from the shock cone is clearly seen in the $\mathrm{O}$ VI greyscale plot presented in Figure 1 as a bright feature, moving from the red to 


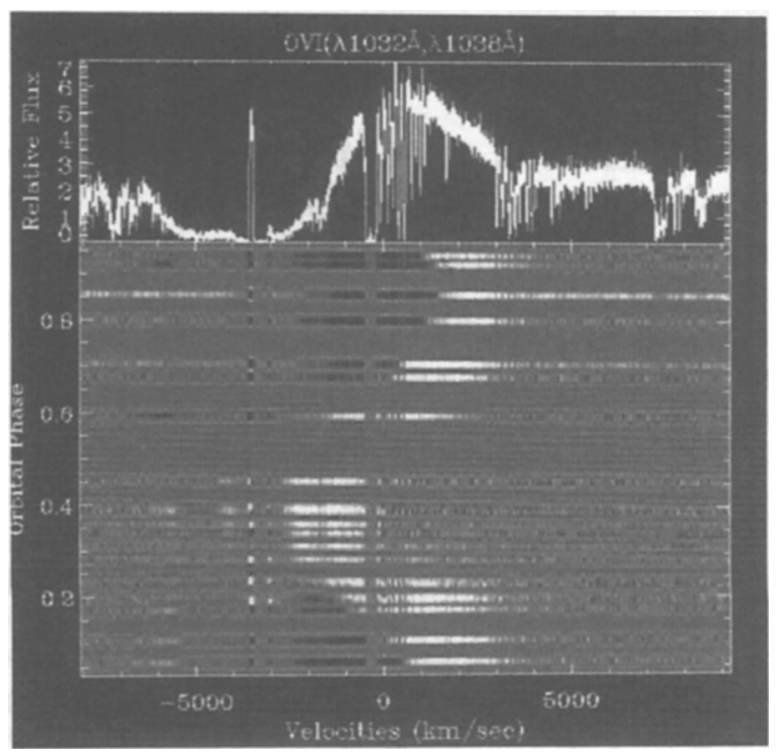

Figure 1. Greyscale plot for $\mathrm{O}$ VI $\lambda 1032 / 1038$ of the differences between individual spectra and the reference spectrum plotted at the top.

the blue from phase 0 (WR in front) to 0.5 , and back again from 0.5 to 0 . The figure presents differences between individual spectra and the reference spectrum plotted above, as a function of orbital phase (orbit from Moffat et al. 1990).

Atmospheric eclipses are clearly visible in Figure 1 as a dark feature with a decreasing width from phase 0 to 0.5 , as expected from the system geometry. The $\mathrm{CIV}+\mathrm{C}$ III line also shows this behaviour, but an additional emission moving in anti-phase with the excess shock emission is present with a central velocity of $-2700 \mathrm{~km} \mathrm{~s}^{-1}$ if due to C III, or $-1000 \mathrm{~km} \mathrm{~s}^{-1}$ if from C IV and an amplitude of $1000 \mathrm{~km} \mathrm{~s}^{-1}$. We have yet to identify the precise origin of this feature.

From the equations derived by Lührs (1997):

$\mathrm{FWHM}_{\mathrm{ex}}=C_{1}+2 v_{\mathrm{str}} \sin \theta \sqrt{1-\sin ^{2} i \cos ^{2} \phi}, \quad$ and $\quad \mathrm{RV}_{\mathrm{ex}}=C_{2}+v_{\mathrm{str}} \cos \theta \sin i \cos \phi$ which describe the displacement $\left(\mathrm{RV}_{\mathrm{ex}}\right.$, these data) and width variations (FWHM $_{\text {ex }}$, from the eclipse-free CIV $\lambda 5808$ line in Bartzakos et al. 2001) of the excess shock emission, we estimate that the shock opening angle $\theta=120^{\circ}$. This agrees very well with the value of $122^{\circ}$ calculated with the equation of Usov $(1992)\left(\theta\left(^{\circ}\right)=120\left(1-\eta^{\frac{2}{5}} / 4\right) \eta^{\frac{1}{3}}\right)$, where $\eta$ is the momentum ratio of the two winds.

\section{References}

Bartzakos, P., Moffat, A.F.J., Niemela, V.S. 2001, MNRAS 324, 33

Lührs, S. 1997, PASP 109, 504

Moffat, A.F.J., Niemela, V.S., Marraco, H.G. 1990, ApJ 348, 471

Usov, V.V. 1992, ApJ 389, 635 